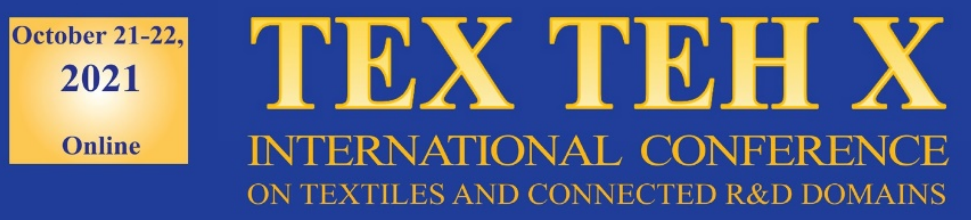

\title{
INTERACTION OF CLOTHING IN THERMOREGULATION IN THE CASE OF PRETERM INFANTS
}

DOI: $10.35530 /$ TT.2021.12

\author{
V. Danila ${ }^{1 *}$, A. Curteza ${ }^{1}$, S. Balan ${ }^{2}$ \\ ${ }^{1}$ Technical University „Gheorghe Asachi” Iasi, Romania, Faculty of Industrial Design and Business \\ Management, Iasi, Romania \\ (E-mail: victoriavasiledanila@gmail.com, acurteza@gmail.com) \\ ${ }^{2}$ Technical University of Moldova, Faculty of Textile and Polygraphy, Department of Design and \\ Technologies in Textile and Polygraphy, Chisinau, Republic of Moldova \\ (E-mail: stela.balan@adm.utm.md)
}

\begin{abstract}
The paper presents a study about the thermoregulation in the case of premature babies and the importance and influence of their clothing in this complex process. The temperature of premature babies has to be between $36.5-37.5^{\circ} \mathrm{C}$ but, hypothermia (axillary temperature $<36.5^{\circ} \mathrm{C}$ ) is a common situation. This is primarily due to a large surface area and metabolic mass ratio that results in heat loss. Hypothermia among new-borns is considered an important contributor to neonatal morbidity and mortality. In this context, it has been identified that clothing products are recommended to maintain the optimal body temperature for those born prematurely. The elaborate clothing products aim at maintaining a thermal comfort and certain physiological indicators. Skin temperature and tactile sensations also play an important role in the growth and development of premature babies. In this regard, 15 premature infants were used as study subjects and their temperature profile was recorded. The proposed clothes aimed to maintain the baby's temperature in the normal range and provide a pleasant aesthetic appearance, while helping to improve the medical manipulations to which these children are subjected.
\end{abstract}

Keywords: body temperature, thermoregulation, clothing, comfort, premature babies

\section{INTRODUCTION}

The World Health Organization (WHO) has recognized neonatal hypothermia as a contributing factor to the risk of morbidity and mortality in newborns. Hypothermia was defined by the WHO as body temperature below normal $\left(36.5^{\circ} \mathrm{C}-37.5^{\circ} \mathrm{C}\right)$ and was subclassified into three grades: mild $\left(36.0^{\circ} \mathrm{C}-36.5^{\circ} \mathrm{C}\right)$, moderate $\left(32.0^{\circ} \mathrm{C}-35.9^{\circ} \mathrm{C}\right)$ and severe hypothermia $\left(<32.0^{\circ} \mathrm{C}\right)$ [1]. For each of these classifications, there are guidelines in place to respond to or manage hypothermia [2]. Furthermore, the WHO has published guidelines on thermal care and has included thermal care for newborns as one of the essential elements of essential care for newborns (ENC) that should be provided to all newborns, regardless of how the temperature is taken [3].

The American Academy of Pediatrics (PAA) [4] and the American College of Obstetricians and Gynecologists (ACOG) (1997) [5] and the World Health Organization (WHO) [1] define normal axillary temperatures to be between $36.5^{\circ} \mathrm{C}$ and $37.5^{\circ} \mathrm{C}$. 


\section{NEONATAL THERMOREGULATION}

Hypothermia occurs when the axillary temperature of the newborn drops below $36.3^{\circ} \mathrm{C}$ [5] or below $36.5^{\circ} \mathrm{C}[1,4]$. We highlight the following characteristics of preterm infants with a higher risk of heat loss:

- A high ratio between surface and body

- Decreased subcutaneous fat

- Higher water content for the body

- Immature skin that leads to increased water by evaporation and heat loss

- Poorly developed metabolic mechanism to respond to thermal stress (eg no tremors)

- Altered blood flow (eg peripheral cyanosis) [1,6-8].

Premature neonates are at an increased risk of developing hypothermia and are more vulnerable to cold stress than the term ones. The axillary temperature (figure 1) should be monitored within the first 30 minutes of life, and then every 1 hour, and should be maintained between $97.7^{\circ} \mathrm{F}$ and $99.5^{\circ} \mathrm{F}\left(36.5^{\circ} \mathrm{C}\right.$ and $\left.37.5^{\circ} \mathrm{C}\right)$ [9].

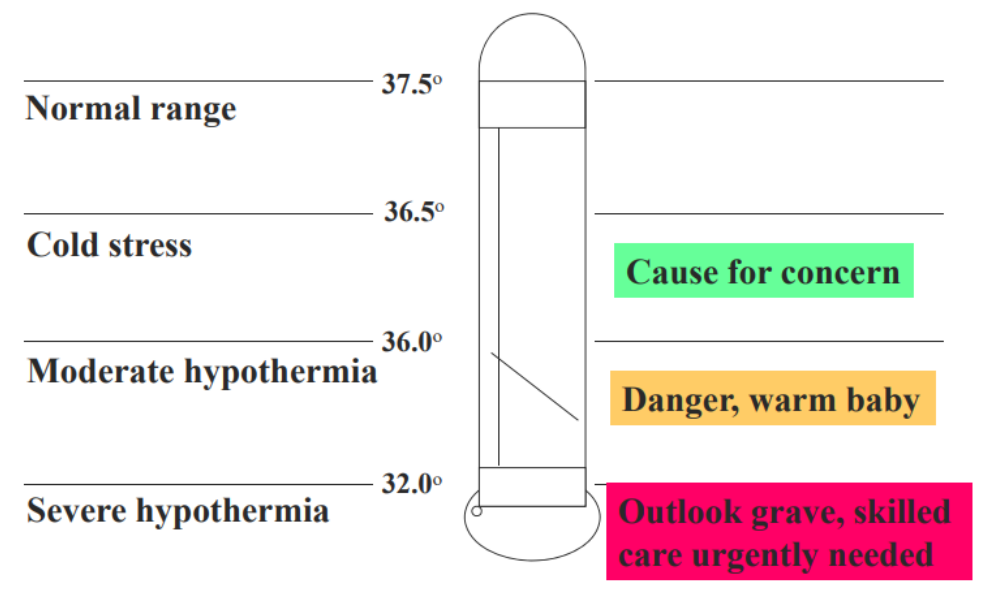

Figure 1. Newborn temperature $\left({ }^{\circ} \mathrm{C}\right)$

Early clinical signs which should arouse suspicion of cold stress due to hypothermia are: the feet are cold to the touch; weak sucking ability - inability to nurse; reduction in activity - lethargy; and a weak cry [10]. Thermoregulation is a biological priority for all homeothermic species [11]. New-borns, particularly preterm and low-birth weight (LBW) infants, have limited capacity for thermoregulation during the first weeks of life. The optimal environmental temperature is named thermal neutral temperature, at which metabolic requirements of the organism are minimal [12,13]. Both a decreased and an increased core temperature increase the metabolic rate of newborns, [13] who have only very limited ability to maintain a normal temperature and easily become hypothermic or hyperthermic (tables 1 and 2).

Table 1. Normal human body temperature and hypothermia [1]

\begin{tabular}{|c|c|}
\hline Description & Range \\
\hline Normal & $36.5-37.5^{\circ} \mathrm{C}$ \\
\hline Cold Stress Moderate & $36.5-36^{\circ} \mathrm{C}$ \\
\hline Hypothermia & $36-32^{\circ} \mathrm{C}$ \\
\hline Severe Hypothermia & $<32^{\circ} \mathrm{C}$ \\
\hline
\end{tabular}


Table 2. Risk factors of prematurity for hypothermia [14-16]

\begin{tabular}{|c|l|}
\hline No. & \multicolumn{1}{|c|}{ Risk factors } \\
\hline 1 & Increased surface area to body mass ratio \\
\hline 2 & Decreased subcutaneous and brown fat \\
\hline 3 & Greater body water content \\
\hline 4 & Increased evaporative water and heat losses due to immature skin barrier \\
\hline 5 & Decreased body tone \\
\hline 6 & Immature thermoregulatory system \\
\hline
\end{tabular}

Below we present ways to lose body heat: evaporation, conduction, convection and radiation (figure 2 ).

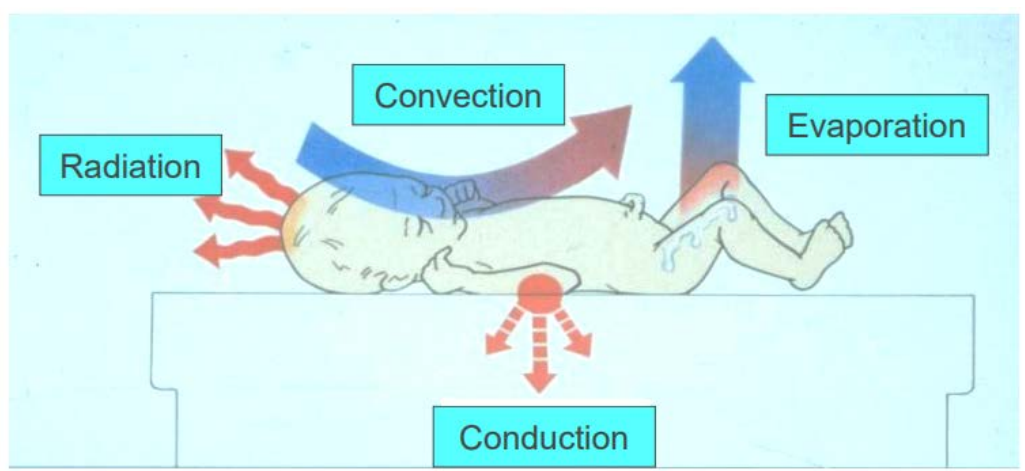

Figure 2. Ways of heat loss [13]

\section{THERMAL SUPPORT OF MODERATELY PREMATURE NEONATES}

From the above discussion one can realize the importance of providing thermal regulation that supports the immature structural and functional integrity of premature neonates. Thermal regulation is important because heat production requires oxygen consumption and energy use:

Persistent hypothermia (table 3) may deplete energy stores impairing weight gain. The following section will present the study of interventions that mainly reduce premature neonates heat lost by the four modes (table 2) and support the conservation of energy balance [17].

\subsection{Measures to prevent hypothermia}

The main measures that may prevent hypothermia include:

1. Increase the room/delivery room temperature $>25^{\circ} \mathrm{C}\left(77^{\circ} \mathrm{F}\right)$.

2. Use of radiant warmer for resuscitation if available.

3. Use of chemical warming mattress.

4. Use of occlusive/polyethylene wrap or large plastic bag (i.e. gallon size food grade storage bag) for neonates $<28-30$ weeks' gestation.

5. Use of knit hat.

6. Neonates born at more than 32 weeks gestation may be placed skin-to-skin with mother, if stable.

7. To avoid burns, hot water bottles or gloves filled with hot water, to warm the neonate, are forbiden [18]. 


\subsection{Materials and methods}

The aim of this study was to maintain the warmth of premature and low birth weight babies, to improve infant state and to determine the safety and efficacy of designed clothing products. The study took place inside the Gh. Paladi or Chisinau hospital, where the medical histories of premature babies were studied. During the study were present medical sisters and doctors responsible for the given department. Premature babies were dressed in ordinary products and products of appropriate size (body and pants, socks, gloves, hat) were subsequently monitored to monitor body temperature. The time interval was 15 minutes at the first measurement of body temperature after every 30 minutes for 24 hours. The data were collected in subsequently processed documents.

Local reactions, if any, due to the texture and quality of the textile material that come in contact with the baby's skin, have also been closely monitored.

We observed 15 premature and low birth weight infants who were hospitalized in the NICU and were kept in an incubator or under radiant heating conditions. Each child's temperature started at $36.5^{\circ} \mathrm{C}-37.5^{\circ} \mathrm{C}$. These infants were constantly monitored for their temperature profile. The average weight of these infants was $1.65 \mathrm{~kg}$, and the average gestational age was 34.75 weeks.

\subsection{Results}

The data of the study were collected in the form presented in table 4, which shows the values of body temperature of children dressed in different products. From the analysis of these data we conclude that children who have been dressed in morphometrically adapted products show an insignificant decrease in body temperature.

Table 3. Study sample

\begin{tabular}{|c|c|c|c|c|c|c|c|c|c|c|}
\hline $\begin{array}{c}\text { Time, } \\
\text { min/no. }\end{array}$ & $\mathbf{0}$ & $\mathbf{1 5}$ & $\mathbf{3 0}$ & $\mathbf{4 5}$ & $\mathbf{6 0}$ & $\mathbf{9 0}$ & $\mathbf{1 2 0}$ & $\mathbf{1 5 0}$ & $\mathbf{1 8 0}$ & $\mathbf{2 1 0}$ \\
\hline $\mathbf{1}$ & 37 & 37.3 & 36.8 & 37.2 & 37 & 36.5 & 36.9 & 37 & 37.2 & 36.8 \\
\hline $\mathbf{2}$ & 36.6 & 37.4 & 37.3 & 37.2 & 37 & 36.6 & 36.4 & 37.3 & 37.1 & 37 \\
\hline $\mathbf{3}$ & 36.6 & 37.5 & 37.6 & 37.2 & 37 & 37.4 & 37 & 36.5 & - & - \\
\hline $\mathbf{4}$ & 36.6 & 37.6 & 37.8 & 37.3 & 37.4 & 37.2 & 37.3 & 36.4 & 36.7 & 37 \\
\hline $\mathbf{5}$ & 36.8 & 37.3 & 37.5 & 37.3 & 36.7 & 36.8 & 37 & 37.2 & 36.3 & 36.6 \\
\hline $\mathbf{6}$ & 37.2 & 36.8 & 37.3 & 37.6 & 37.2 & 36.6 & 37 & 36.5 & 37.2 & 36.5 \\
\hline $\mathbf{7}$ & 36.8 & 37 & 37.4 & 36.9 & 37 & 37 & 37.5 & 37 & 37.4 & 36.5 \\
\hline $\mathbf{8}$ & 36.8 & 37.5 & 37.6 & 37.4 & 37 & 37.4 & 37 & 36.5 & - & - \\
\hline $\mathbf{9}$ & 36.9 & 37.6 & 37.8 & 37.6 & 37.4 & 37.2 & 37.3 & 36.4 & 36.7 & 36.5 \\
\hline $\mathbf{1 0}$ & 37 & 37.3 & 36.8 & 37.2 & 37 & 36.5 & 36.9 & 37 & 37.2 & 37 \\
\hline $\mathbf{1 1}$ & 36.8 & 37.4 & 37.3 & 37.2 & 37 & 36.6 & 36.4 & 37.3 & 37.1 & 37 \\
\hline $\mathbf{1 2}$ & 36.7 & 37.5 & 37.6 & 37.2 & 37 & 37.4 & 37 & 36.5 & - & - \\
\hline $\mathbf{1 3}$ & 36.9 & 37.6 & 37.8 & 37.3 & 37.4 & 37.2 & 37.3 & 36.4 & 36.7 & 36.5 \\
\hline $\mathbf{1 4}$ & 37 & 37.3 & 37.5 & 37.3 & 36.7 & 36.8 & 37 & 37.2 & 36.3 & 36.5 \\
\hline $\mathbf{1 5}$ & 36.8 & 37.6 & 37.1 & 36.8 & 37.2 & 37 & 36.6 & 36.9 & 36.8 & 37 \\
\hline
\end{tabular}

\section{CLOTHING INFLUENCE ON THERMOREGULATION}

Temperature regulation is an important function for homeothermic organisms, because body temperature ensures the balance of metabolic chemical reactions and its optimal overall state. The study [19] focused on aspects of thermoregulation at the birth of 
VLBW (small children with birth weight), including epidermal water loss and temperature control, during stabilization and on admission to the neonatal intensive care unit.

Clothing plays an important role in this context. It is known that clothing products form a microenvironment, an insulating layer with properties to reduce the degree of heat loss by evaporation. The developed clothing products aim at increasing the thermal insulation, offering psychosensory and thermal comfort to the child. Therefore, one of the benefits of manufactured products is increased functionality and reduced heat loss.

We took also into consideration that advances in textile research have identified that thermoregulation can be improved through the use of materials with hygienic, aesthetic and physical-mechanical properties. The materials used to make these products were those with the fibrous composition of natural fibers (organic cotton, flax, lyocel). The materials have high hygienic properties, are soft and allow the exchange of heat and humidity with the environment.

In the development of the functional clothes (figure 3) some important aspects were taken into account, such as:

- infants have a large body surface area in relation to their weight;

- the ratio between surface and body weight is two to three times higher than in adults;

- the head of an infant is large regarding the proportion of the body;

- in the case of newborns, up to $75 \%$ of the body heat loss may be from an uncovered head;

- premature and low birth weight babies have less subcutaneous fat for insulation and thus lose heat more easily;

- because babies do not have body fat, they cannot regulate their body temperature; prolonged exposure to environmental factors outside the incubator cools babies' bodies.

The given products are qualitative; they correspond to all the clothing requirements for premature babies, which take into account their degree of development and needs, in order to allow them an easy and comfortable growth. Thus we satisfy the mothers and the medical staff with hygienic, aesthetic products and adequate given situation.
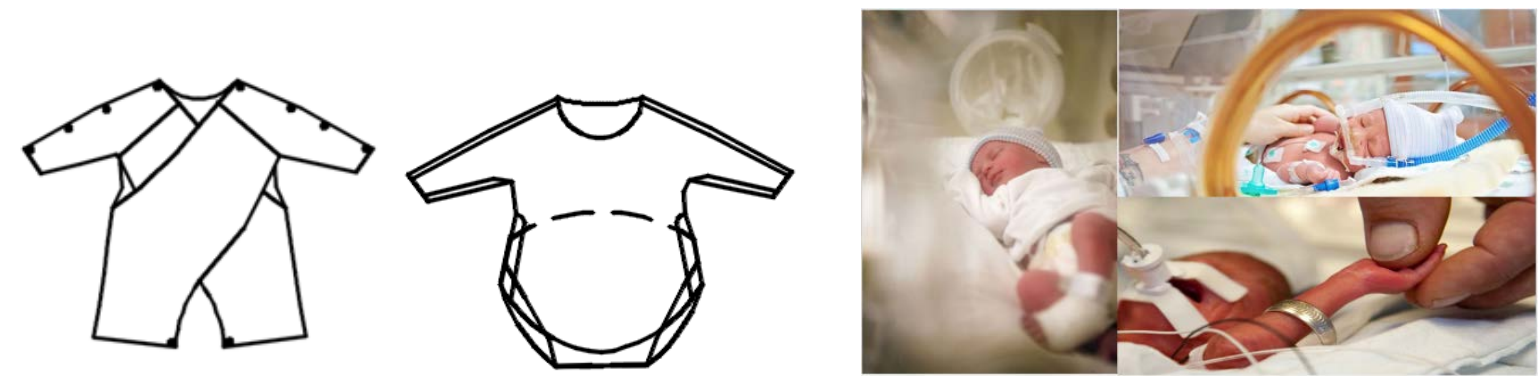

Figure 3. Functional clothes for premature babies

The shape of the product is obtained by winding, the closing system is on the upper and inner edges, with buttons, and has no contact with the child's body, and the product has holes for: head, upper and lower limbs.

Thermal comfort is ensured by the constructive and technological solution proposed for premature babies. The anthropomorphological adaptation of the products to the requirements and needs of premature babies for static and dynamic positions, offers the possibility to provide medical help in a short time. 


\section{CONCLUSION}

The proposed solution is one of the strategies applied in order to increase the efforts of medical institutions as well as of parents whose final goal is to improve the chances of survival and rehabilitation of preterm babies.

Particularities of the premature child's physical development, including the thermoregulation system, determine the hygienic and thermal insulation requirements imposed on clothing products.

This specific problem is behind ongoing efforts to find solutions to eliminate hypothermia in the case of premature infants. One of the issues addressed is the use of appropriate clothing products for premature babies. The products offer thermal insulation and convenience when performing various medical movements and manipulations.

\section{REFERENCES}

[1] World Health Organization, Thermal protection of the newborn: A practical guide, Geneva, 1997, Report No.: WHO/RHT/MSM/97.2

[2] World Health Organization, Pregnancy, childbirth, postpartum, and neonatal care: A guide for essential practice, Geneva, 2007

[3]World Health Organization, Essential newborn care, 1996, Report No.: WHO/FRH/MSM/96.13

[4] American Academy of Pediatrics (AAP), Available at: www.aap.org [Accessed on June 2021]

[5] American Academy of Pediatrics \& American College of Obstetricians and Gynecologists, , Acute care of at-risk newborns: A resource and learning tool for health care professionals, ACoRN Editorial Board, Vancouver, 2012

[6] Blackburn, S.T., Maternal, fetal, \& neonatal physiology: A clinical perspective, 3rd ed., St. Louis: Saunders Elsevier, 2007

[7] Hackman, P., Recognizing and understanding the cold-stressed term infant, In: Neonatal Network, 2001, 20, 8, 35-41

[8] Galligan, M., Proposed guidelines for skin-to-skin treatment of neonatal hypothermia, In: American Journal of Maternal/Child Nursing, 2006, 31, 5, 298-304

[9] Pejaver, Dr. Ranjan K., Interview on Infant Hypothermia, Personal interview, June-July, 2012

[10] World Health Organization, Thermal control of the newborn: a practical guide, 1993, Available at: https://apps.who.int/iris/handle/10665/60042 [accessed on June 2021]

[11] Hey, E., Thermal neutrality, In: Br Med Bull, 1975, 31, 69-74

[12] Baumgart, S., Iatrogenic hyperthermia and hypothermia in the neonate, In: Clin Perinatol, 2008, 35, 183-197, ix-X

[13] Sherman, T.I., Greenspan, J.S., St. Clair, N., Touch, S.M., Shaffer, T.H., Optimizing the neonatal thermal environment, In: Neonatal Newt, 2006, 25, 251-260

[14] Aylott, M., The neonatal energy triangle. Part2: Thermoregulatory and respiratory adaption, In: Paediatric Nursing, 2006, 18, 7, 38-42

[15] Knobel, R., Holditch-Davis, D., Thermoregulation and heat loss prevention after birth and during neonatal intensive-care unit stabilization of extremely low-birthweight infants, In: Journal of Obstetric, Gynecologic, and Neonatal Nursing: JOGNN / NAACOG, 2007, 36, 3, 280-287

[16] Sedin, G., Agren, J., Water and heat--the priority for the newborn infant, In: Upsala Journal of Medical Sciences, 2006, 111, 1, 45-59

[17] Lewis, L.A., Nursing care procedures, thermal regulation and growth of the moderately premature neonate in the neonatal intensive care unit, Kent State University College of Nursing, Dissertation thesis, 2014, 128

[18] Illinois ESF-8 Plan: Paediatric and Neonatal Surge Annex Pediatric and Neonatal Care Guidelines, 2017, June 
[19] Danila, V., Curteza, A., Thermoregulatory clothes for premature babies: effectiveness and risks, In: Proceedings 17th Romanian Textiles and Leather Conference - CORTEP 2018, Iasi, 7-9 November 2018, ISSN-L 2285-5378, 95-100 